\title{
DONDE LA CIUDAD SE ESCRIBE. PRENSA, URBANIZACIÓN Y CULTURA EN ROBERT E. PARK ${ }^{1}$ WHERE THE CITY IS WRITTEN. ROBERT E. PARK: ON PRESS, URBAN GROWTH AND CULTURE
}

\author{
Emilio M. Martínez Gutiérrez \\ Universidad Complutense de Madrid, España \\ emilmmar@ucm.es
}

\begin{abstract}
Cómo citar / Citation
Martínez Gutiérrez, Emilio M. (2016). "Donde la ciudad se escribe. Prensa, urbanización y cultura en Robert E. Park". OBETS. Revista de Ciencias Sociales, 11(2): 487-512. doi:10.14198/OBETS2016.11.2.05
\end{abstract}

\section{Resumen}

La recepción de la obra de Park se ha ceñido principalmente a sus trabajos de ecología urbana. Hoy día se advierte una lectura más amplia y plural que explora entre otros aspectos sus aportaciones teóricas y empíricas a otros campos de interés como la opinión pública y la prensa. El artículo aborda la vinculación existente entre prensa y ciudad, laboratorios de la modernidad y espacios de socialización, en el pensamiento de Park, partiendo de su experiencia como reporter y su vocación académica.

Palabras clave: Investigación sociológica/ Comunicación/ Prensa/ Ciudad/ Park/ Escuela de Chicago/ Cultura urbana.

\section{Abstract}

Perspectives on reception of R. Park's work have mainly revolved around urban ecology. At present we can perceive a broad and diverse reading that explores their theoretical and empirical contributions to other subjects such as public opinion and press and so on. This article deals with the link between press

\footnotetext{
${ }^{1}$ Este artículo fue escrito en el marco de una investigación de mayor alcance sufragada por el Ministerio de Educación dentro del Programa de movilidad de investigadores 2015 (PRX 14/00525).
} 
and city, laboratories of modernity and spaces of socialization, in the work and life of Park, reporter and sociologist.

Key words: Sociological Research/ Communication/ Press/ City/ Park/ Chicago School/ Urban Culture

\section{Extended Abstract}

Since the publication of "The city: Suggestion for the Investigation of Human Behaviour in the Urban Environment", first in 1915 (American Journal of Sociology) and then -with significant variations- in the collective volume The City, written with Ernst Burgess, Robert McKenzie and Louis Wirth, the reception of the Robert Ezra Park's work has essentially adhered to the urban dimension. In detail to that original scientific perspective known as urban ecology (one of the most important contributions of the Chicago School). In spite of the obvious merits, we can assure that this research line is only a bright stage of a long career related with the specific processes of the American urbanization. This partial reading, predominant until the 1990s, begins recently to be revised, either exploring the course of a comprehensive understanding of the overall work or examining other issues such as racial issues, immigration, public opinion and sociology of press. Of course, the issues and realities referred appear plentiful and crosswise in many writings of Park. Cause of these issues integrate the central problematic that gives full meaning to intellectual progression of R. Park: the study of social interactions, or the compositions of social order in changing times. Both in the one as in the other matter social communication plays a key role.

The aim of this paper is to uncover the multidimensional conception of social order and social change at Park's thought such as shown in the study of the city like ecological pattern and moral order in relation to the social communication and press. In this sense, we aspire to know the connexion between Park as author dedicated to journalism and that becomes sociologist and scholar of urban research and sociology of the press. What does the path of the journalist researcher in urban sociology? What influence does the world of news and communication in the constitution of metropolitan fabric and social structure? What are the consequences for press of heterogeneous population growth in cities? We seek to establish the profiles of this connections using as the most appropriate methodology the review of his work, following a kind of hermeneutic cycle based on the interpretation of texts in contexts. According to this methodology we can notice a plural and transversal interpretation of Robert Park's work, after considering inter alia the historical, social and intellectual context of their works and life (the reform discussion, the immigrant question in war times and so on). Struck by the dynamism that has regained its contribution to the consideration of public opinion, the social communication and the press (an absolutely modern interpretation). Such recovery rectifies certainly the canonical reading whereby the first incursion of sociological research in this sphere of studies corresponded to the work of Columbia University. But especially it involves the recognition of research consistency of the author. Indeed, without this reference to that plane of Park's 
path it would be very difficult to scope a full understanding of its sociological conception.

Until Park, Sociology generally had neglected the study of the press. This omission was certainly strange as press was a modern cultural product. Only in Max Weber's speech to the German Sociological Association (1910) we found a serious solicitation to exploring this social domain away from a moralistic approach. That is precisely the survey we find in Park, within a comprehensive program of work powered by two sources: (a) the experience as a reporter, editor and publicist; and (b) the intellectual reflection. The practice becomes an object of study. Then, if Park is not a pioneer, we can consider him a qualified analyst.

Park's prospect on press runs between intuitive knowledge and scientific knowledge, according to the distinction exposed in "News as a Form of Knowledge" (1940). There are several texts about this topic: "Foreign Language Press and Social Progress" (1920), "The Natural History of the Newspaper" (1923), "American Newspaper Literature" (1927), "News and the Human Interest Story" (1940), "News and the Power of Press" (1941), "Moral and the News (1941). We can observe that this course is more analytical than critical, taking the role of information in democracy and collective action (in the perspective of political sociology). This perspective derives in part from the Anglo-Saxon interpretation about the role of press as control device of citizenship (training / information / concerted action) and Government. It is a very interesting line of work that opens on the professional group: the role of the reporter and the course of their information. Another very original path is the one linked to the study of the urban phenomenon, which stems largely from his activity as city reporter and city editor. We find here how Park understands press and city as laboratories of modernity, and press becomes, like naturalistic romances, the privileged place to encrypt the urban daily life.

Sociologist imagined by Park seems a super-reporter. There is not an epistemological discontinuity between journalistic and sociological praxis at Park's thought, only a difference of degree. However, the journalistic experience of Park takes curious paths on the issues of sociological research and explains its obstinacy for direct data collection and the subjects themselves. Salerno (Sociology Noir. Studies at the University of Chicago in Loneliness, Marginality and Deviance 1915-1935, 2007) suggest the precipitation of a kind of sociology noir. Park's research explores the link between communication, press and city in several texts: "Natural History of Newspapers' (1923)," The immigrant community and immigrant press "(1925)," Urbanization as Measured by newspaper circulation "(1929) "Newspaper circulation and metropolitan regions" (1933). We are not talking about something cross, but the forthright study of the relation (1) between press and urban evolution; and (2) between press and urban culture. Indeed, "The City" (1915/1925) -text that opens the urban sociology research-is inconceivable without this reference to communicative processes. This connection is also expressed in many other "urban" texts on communication and media in the hardware configuration of the city or those where the author examines the role of the press on modern urban 
culture. Because urban growth is never seen as simple agglomeration of activities, social groups and individuals but as a cultural process. The modern press, a product of these urban conditions, contributes to the spread of urban values and also speeding up the assimilation process of city inhabitants of all conditions and origins. Hence, it is granted a key role in delineating a new social order, typical of complex environments; and moreover, a socializing role.

\section{INTRODUCCIÓN}

En 1915 vio la luz en el American Journal of Sociology el texto "La ciudad. Sugerencias para el análisis del comportamiento humano en el medio urbano", artículo firmado por Robert E. Park. Una segunda versión aparecería con algunos cambios diez años después en una obra colectiva (The City) donde bajo la dirección de Park participaban investigadores como Burgess, McKenzie y Wirth, esto es, el núcleo duro de lo que después sería conocido como la ecología urbana de Chicago.

Inmediatamente desde su edición hasta hoy la obra ha resultado un referente para la investigación sociológica sobre la ciudad. Pese a las controversias suscitadas respecto al valor inaugural que tendría para la sociología urbana y al alcance teórico que se le podría conceder, en el ámbito académico se admite que la recepción de la obra de Park se ha ceñido fundamentalmente a la dimensión urbana, en concreto a esa original perspectiva sobre la articulación de las comunidades en el territorio conocida como ecología de la ciudad.

Ciertamente el conjunto de sus escritos sobre el fenómeno urbano destaca por su lucidez y agilidad, con una orientación positiva hacia el hallazgo, paralela a una menor preocupación por la conceptualización. Pero con todo, y sin restarle sus enormes méritos, no deja de ser sino una fase brillante de una trayectoria dilatada, relativa, de un lado, a un periodo concreto de su producción y, de otro, a una etapa igualmente especifica del proceso de urbanización de la sociedad americana. Podríamos decir que resulta pues una lectura parcial. Predominante durante muchas décadas, esta interpretación comienza a ser rectificada de unos años a esta parte, bien explorando el cauce de una comprensión global del conjunto de su obra (Abbot, 1999; Guth, 2008), bien discurriendo por otros ámbitos característicos de su trabajo relativamente acotados, como los problemas raciales, la inmigración, la opinión pública y el mundo de la prensa. Relativamente acotados, insistimos, pues los temas y las realidades a que se refieren aparecen abundante y transversalmente en muchos de sus escritos cuando la cuestión central interpelada lo consiente. E integran esa problemática que da sentido a su progresión intelectual: el estudio de las interacciones 
sociales y el problema del orden social en situaciones de cambio acelerado. En lo uno y en lo otro la comunicación social desempeña un rol fundamental.

\section{OBJETIVOS Y METODOLOGÍA}

A partir del examen de los escritos y trayectoria de Park, siguiendo una hermenéutica que interprete la relación entre textos y contextos, el objetivo que proponemos en este trabajo es abordar la concepción poliédrica de lo social característica de su pensamiento, tal como se presenta en el estudio de la ciudad como modelo ecológico y orden moral en relación con la comunicación social y la prensa. En ese sentido, planteamos la correspondencia entre el Park dedicado al periodismo y el que deviene sociólogo y académico, estudioso de la ciudad y de la prensa. ¿Qué aporta la trayectoria del periodista al del investigador en sociología urbana? ¿Qué influencia tiene el mundo de las noticias y de la comunicación en la constitución del entramado físico y social metropolitano? ¿Qué evolución depara a la prensa la acumulación de población heterogénea en las ciudades? ¿No es la prensa el primer lugar donde la ciudad se cuenta, como sostiene Muhlmann (2008)?

Estas cuestiones sólo pueden enfrentarse sobre la base de aceptar que Park no se deja encerrar "en lecturas simplificadoras ni entre imperativos binarios" (Plenel 2008: 8). Con él siempre deambulamos por ámbitos in fieri, hibridados, acordes al itinerario de quien nunca dejó de ser "a Minnesota boy", como lo describió Raushenbush (1979) en uno de los estudios más completos dedicados al autor. Un tipo de la frontera intermedia, lo que deja impronta en su andadura y disposición -de un sitio a otro, de una ciudad a otra, de un oficio a otro-. Guth (2012) también se refiere a él en términos muy parecidos, cuando describe sus pasos por Berlín, Estrasburgo y Heidelberg: un Wandervogel [ave de paso] cuya existencia nómada se acomoda a su más que notable vagabundeo intelectual. Esa disposición explica en parte su talento para franquear territorios al margen, lugares intersticiales, sujetos y vidas en trance, atento a la hibridación cultural como la que representa la ciudad pero igualmente la prensa, a la vez instrumento de control e integración social, mercancía y producto cultural, espacio de encuentro y de socialización.

\section{LA RECEPCIÓN CONTEMPORÁNEA: LECTURAS PLURALES}

En la reciente sociología francesa se manifiesta la voluntad de superar la estricta acotación de la obra parkiana al ámbito urbano. Dicha lectura procedía del "descubrimiento" de la escuela de Chicago por Chombart de Lauwe (1950) has- 
ta su reactualización por parte de Grafmeyer \& Joseph (1979) ${ }^{2}$. Los trabajos de J. M. Chapoulie (2001), P. Lannoy (2004), D. Cerfaï (2008), D. Le Breton (2008), y muy en especial de Suzie Guth (2004, 2008), entre otros, aconsejan una interpretación más amplia, plural y transversal de su obra, atendiendo entre otros aspectos al contexto histórico, social e intelectual de su trabajo (el debate reformista, su inserción en el pragmatismo, la prolongación en el interaccionismo simbólico, las derivaciones por la sociología política hasta su recepción por disciplinas concurrentes en el estudio de la sociedad). Llama la atención el dinamismo con que se ha recobrado su aportación al estudio de la opinión pública, la comunicación social y la prensa (una interpretación absolutamente moderna). Tal recuperación rectifica sin duda la lectura canónica según la cual la primera incursión de la investigación sociológica en este campo de estudios correspondía a los trabajos de Columbia. Pero especialmente supone el reconocimiento de la coherencia investigadora del autor. En efecto, sin la referencia a esa faceta de la trayectoria parkiana sería harto difícil alcanzar una comprensión cabal del pensamiento y orientación de sus exploraciones sociológicas.

Una primera aproximación en esta dirección lo encontramos en el estudio de Bourmeau (1988), que tienen continuidad en los trabajos de Muhlmann en Du journalisme en démocratie (2004). En esta obra se dedica todo un capítulo a la sociología de la prensa en Park. Por su parte, Suzie Guth (2008) retoma los planteamientos sobre la muchedumbre y la opinión pública expuestos en la tesis doctoral de Park, Masse und Publikum (texto traducido al francés en el 2008). Y en Le journaliste et le sociologue (2008) Muhlmann y Plenel reúnen algunos textos de Park consagrados al estudio de las noticias, la circula-

${ }^{2}$ En realidad la primera referencia en el ámbito académico francés a la escuela de Chicago la encontramos en el texto "Chicago, expérience ethnique" (1932) de Maurice Halbwachs. Éste realizó una estancia en la Universidad de Chicago durante el otoño de 1930, por invitación de E. Faris. En el citado texto se da cuenta de los trabajos, los planteamientos y el contexto de esa "escuela particular de sociología" como la denominó el durkheimiano. De la realidad social de Chicago, Halbwachs da testimonio también en la serie de ocho artículos publicados durante ese tiempo en el diario Le Progrès de Lyon, lo que no deja de tener su interés en el marco de las relaciones entre la sociología (científica) y la información periodística.

Merece la pena referir el primer contacto entre Park y Halbwachs en la Universidad de Chicago, no muy afortunado. En una de las cartas que dirige a su mujer Halbwachs le confiesa que: "Hoy, cuando me disponía a comer solo, un sociólogo llamado Park ha venido a sentarse a mi mesa. Este Park es un tipo que debe rondar los sesenta, de fisionomía adusta, pero al que no le falta estilo. Tiene el aire de un filósofo alemán. Pero es uno de los tipos a quien más me cuesta comprender. Se dedica a la sociología urbana. Cuando subí a mi habitación ya no podía más". Topalov ha reunido las cartas, textos y los escritos científicos asociados a la estancia americana de M. Halbwachs en Écrits d'Amerique (2012). 
ción de la prensa, la formación de la opinión pública y su relación con el poder. La aproximación cultural y política de la prensa también es tratada en la exposición de Lazar (2006).

En la sociología española la recepción de Park ha seguido un curso similar, privilegiando la referencia ecológica. Atendiendo a sus derivaciones sobre la desorganización de las comunidades locales, la antropología y el trabajo social han discurrido igualmente por ese ámbito casi estipulado. No obstante, otras lecturas han actualizado su reflexión sobre la comunicación y la prensa. La traducción de La masa y el público apareció en la Revista Española de Investigaciones Sociológicas en 1996, con la excelente presentación de Sánchez de la Yncera y López-Escobar. Poco después se editó la monografía de R. Berganza (2000), hasta hoy el texto más completo en español sobre esta dimensión del pensamiento de Park. En 2013 Cuadernos de Información y Comunicación publicó la traducción del texto de Park "Moral and the News" (1941). No deja de ser interesante que haya sido en el ámbito más hibridado de los estudios de Comunicación, quizás menos inclinado al prejuicio hacia lo que algunos plantean como investigaciones "profanas" sobre la realidad social, donde se ha impulsado una lectura más abierta de la obra de Park.

\section{COMUNICACIÓN Y SOCIOLOGÍA DE LA PRENSA}

Considerando que la prensa puede operar como agente de transformación y control social, y que es a la vez producto de la modernización, como la propia sociología, cabía esperar el interés de ésta por el escrutinio de la prensa. Cierto que contamos con observaciones de Tocqueville, Durkheim o Tarde, pero se trata de reflexiones muy generales, incorporadas o derivadas de sus teorías sobre la democracia, la solidaridad social, las representaciones colectivas, las leyes de la imitación social... en el advenimiento de una sociedad de masas, industrial y urbana. Ninguna aborda específicamente el papel de la prensa aunque si se preocupan ciertamente por la opinión pública. (No deja de sorprender que autores como Marx o Simmel, que tanto escribieron en los periódicos de su tiempo, apenas incorporasen la cuestión a sus reflexiones). El caso de Max Weber es otro. Es bien conocida su contribución a la caracterización de la prensa y del periodismo en el ámbito de las relaciones (y vocaciones) de poder, en El político y el científico. Pero además encontramos su firme invitación al estudio sociológico de la prensa en su alocución ante la Asociación Alemana de Sociología (Frankfurt, 1910). Weber estima necesario alejarse de posiciones críticas de corte moralizante para atender el quehacer de los profesionales de la prensa (periodistas, editores...), los requisitos y las influencias actitudinales, o su papel en la construcción del espacio público... Es precisa- 
mente este escrutinio lo que hallamos en los escritos de Park, donde la invitación se convierte en un firme programa de trabajo. En su caso, un programa alimentado por dos fuentes: (a) su experiencia como reportero, editor y agente de prensa; y (b) su reflexión intelectual. De Park se puede decir que "antes de escribir sobre la prensa, escribía en ella" (Bourmeau 1988: 51), práctica profesional que deviene objeto de estudio. Si Park no es un pionero, sí es un analista doblemente cualificado.

Su interés por el periodismo se manifestó pronto, animado por J. Dewey, de quien asume el valor social de la comunicación y el rol de la prensa como dispositivo de control social e instrumento de integración y preservación cultural. Dewey le animó a estudiar la naturaleza y función del periódico toda vez que Park había mostrado su inclinación actuando como editor de The Argonaut, periódico universitario de Ann Arbor. Tras graduarse (1887), receloso de la vida académica, desconectada a su juicio de la realidad y muy sedentaria, Park ejerce el oficio entre 1877 y 1898 en diarios de Detroit, Minneapolis, Denver, Nueva York y Chicago. En el Minneapolis Journal trabajó como cub reporter y pasa tres años viajando "y viendo la clase de cosas que ve un reportero" (Park 1950: v). En el Detroit Tribune y el Denver Times actúa como city editor. Alcanza La Meca del periodismo, Nueva York, en 1892 e intenta la crítica cultural en el Chicago Journal. Por otro lado, Dewey facilitó su encuentro con Franklin Ford y la aventura del Thought $\mathrm{News}^{3}$, un proyecto fallido de diario preocupado por el registro fiel de las fluctuaciones de la opinión pública, con una información rigurosa de los problemas sociales.

Dejando atrás esta práctica directa (aún actuaría como agente de prensa de la Congo Reform Assocation y del líder negro Booker T. Washington en el Tuskegee Institut) Park inicia el camino de la reflexión intelectual. Su interés por la prensa deriva hacia la reflexión sobre las noticias, la comunicación y la opinión pública. De Harvard pasa a Berlín y a Estrasburgo, donde estudia con Windelband (Escuela neokantiana de Baden), a quien seguirá a Heidelberg, donde defiende su tesis Masse und Publikum. Lo que se pretendía un estudio sobre el periodismo termina orientándose hacia un análisis conceptual, procurando discernir los significados atribuidos a las nociones de masa y de público en un diálogo entre la filosofía americana (Boston y Michigan) y los planteamientos europeos (las tesis de Windelband, Tarde, Le Bon, Durkheim o la escuela italiana de Lombroso).

La perspectiva de Park sobre la prensa discurre entre el conocimiento intuitivo de quien está en el oficio y el conocimiento científico que aspira a disec-

${ }^{3}$ El periódico fue pensado como un instrumento pedagógico destinado a la explicación de la realidad social. Pero también se pretendía una herramienta de cultura cívica (política) para el fortalecimiento de los valores democráticos. 
cionar su naturaleza, haciendo buena esa distinción entre un saber fundado en la familiaridad y un saber objetivo de la ciencia que expondría en "News as a Form of Knowledge" (1940). Son varios los textos consagrados al ámbito de la prensa y del periodismo: "Foreign Language Press and Social Progress" (1920), "The Natural History of the Newspaper" (1923), "American Newspaper Literature" (1927), "News and the Human Interest Story" (1940), "News and the Power of Press" (1941), "Moral and the News (1941). En este haz de trabajos se percibe una clara orientación analítica antes que crítica, realizada desde el interior, atendiendo al papel desempeñado por la información en la democracia y en la acción colectiva (situándose así en la óptica de la sociología política). Este recorte deriva en parte de la tradición anglosajona sobre el papel de la prensa como útil de control social de la ciudadanía (formación/información/acción concertada; paso de la masa al público) y del gobierno. Pero es de gran interés la línea de trabajo que abre sobre el grupo profesional, el rol del reportero así como el análisis del recorrido de su producto, la información. Otra vía muy original es la que se vincula con el estudio del hecho urbano, que proviene en gran medida de su actividad como city reporter y city editor.

Abordaremos aquí la forma como esta práctica y reflexión profesional se vierte en su pensamiento sobre la ciudad y las relaciones sociales en el ámbito urbano; el modo en que presenta a la prensa y a la ciudad como laboratorios de la modernidad; o cómo la prensa deviene, al igual que la novela naturalista, lugar privilegiado para cifrar la vida cotidiana de la ciudad.

\section{CONTRIBUCIÓN A LA INVESTIGACIÓN SOCIAL Y URBANA}

Como ya hemos mostrado en otro lugar (Martínez 2013) los primeros cursos de Park en Chicago atendieron básicamente a las cuestiones raciales, la opinión pública y la masa, el periódico y los social surveys. Precisamente la invitación que William I. Thomas le cursó para integrarse a la disciplina del Departamento de Chicago obedecía a la competencia que Park había mostrado sobre estos temas. El de la ciudad no le era ajeno, por supuesto, pero hasta entonces nada había escrito sobre ella desde una perspectiva estrictamente sociológica. La investigación de Lannoy (2004) muestra cómo en realidad el texto conocido como The City es el resultado del curso dado sobre The Survey, tras una adaptación a la temática del "comportamiento humano en el medio urbano" (como indica el título del artículo mismo).

La adaptación revelaba la orientación empírica ambicionada por la sociología de Chicago: documentación, observación directa e investigación aplicada frente a las tentaciones especulativas de la filosofía social o de la perspectiva sociohistórica. Park consideraba que las ciencias puramente intelectuales co- 
rrían el riesgo de perder el contacto con la realidad, hasta el punto de que los símbolos manejados podrían reducirse a simples juegos dialécticos, propios de la escolástica. Las propias opciones y tradiciones intelectuales (el ascendente del pragmatismo) habilitarían la senda inductiva frente a la sociología de gabinete. Pero la opción experimental respondía asimismo a otras exigencias, de contexto y de vocación. Una era enfrentar la compleja realidad de la ciudad de Chicago, prototipo del crecimiento metropolitano. Otra, atender la importancia de la cuestión social, como se refería entonces el conflicto y las desigualdades sociales inherentes al capitalismo, que Chicago experimenta brutalmente por entonces. Esta cuestión ceñía un ámbito prioritario de estudio e intervención, articulado en el espíritu de reforma que impregnó a la prensa y a la sociología estadounidense: el social survey movement. En esta combinatoria de cuestiones Park encuentra en la redacción de The City una oportunidad para impulsar un amplio proyecto de investigación en cuyo diseño y posterior desarrollo confluyen sus experiencias previas como reporter y city editor.

\subsection{Chicago Lab}

En 1904 Max Weber describía de este modo Chicago:

"Chicago es una de las ciudades más increíbles. Junto al lago hay algunos barrios residenciales, bellos y agradables, por lo general casas de piedra de estilo más duro y pesado; justo detrás, viejas casitas de madera, igual que en Helgoland. Luego están los tenement [casas de vecindad] de los obreros y una absurda suciedad vial; nada de adoquines, unas calles miserables fuera del barrio residencial; el estado de las calles de la city, entre los sky-scrapers [rascacielos] es horripilante (...) Delirante es la mezcla de pueblos: los griegos les limpian las botas a los yanquis por cinco centavos; los alemanes son sus camareros; los irlandeses se ocupan de la política; los italianos se encargan de los trabajos más sucios. Toda la enorme ciudad (más grande que Londres) se parece, a excepción de los barrios residenciales, a una persona a quien le hubieran quitado la piel y cuyas vísceras se vieran trabajar" (Weber 1995: 443-444)

"Delirante mezcla de pueblos." En términos muy parecidos lo expresó años después Halbwachs cuando se refería a Chicago como una "experiencia étnica".

"Ante sus ojos se despliegan (...) nuevas fases de una evolución urbana sin parangón. (...) Todo ello en una aglomeración enorme, con múltiples diferenciaciones según raza, nacionalidad, profesión y nivel social, también según los estilos de vida y las características morales, de tal modo que los medios sociales más diversos se yuxtaponen y se enfrentan a veces sin transición. (...) Grupos desintegrados, grupos en formación, vida colectiva dispersada, concentrada, suspendida y ralentizada, agitada y discordante, de tal modo que las características más anormales aparecen ahí con toda claridad (...) o bajo 
formas que no es posible encontrar en ninguna otra parte." (Halbwachs [1932] 2008: 207-208)

No se trataba sólo de su brutal explosión territorial y demográfica (anexionando terrenos conforme se iba colmando de población inmigrante en busca de las oportunidades que una economía en expansión ofrecía), sino también de su heterogénea composición social y cultural. Chicago seguía siendo una ciudad de frontera, con toda esa variedad de tipos y situaciones sociales que llamó la atención de políticos, reformadores, periodistas, académicos, sindicalistas, etc. Park de hecho toma la ciudad como lugar idóneo para el estudio de la vida social, al entender que "amplifica, despliega y exhibe las más variadas manifestaciones de la naturaleza humana" (Park, 1999:126). La mención a la ciudad como laboratorio social, más que un tropo afortunado, parece apuntar a un doble propósito en su caso:

(1) animar el espiritu de curiosidad sin el cual no es posible ahondar en el conocimiento de los hechos sociales;

(2) en símil clínico, asegurar un saber preciso y objetivo más allá de la mera familiaridad con el entorno.

Porque hay un mundo que también se da por supuesto que debe cuestionarse, de ahí la obediencia a la reclamación de Williams James (On a Certain Blindness in Human Beings) ante la opacidad relativa y la diversidad de la vida social, difícilmente aprehensible en el estricto marco de las categorías positivistas.

\subsection{Investigación periodística y sociología noire}

Como confiesa en sus notas autobiográficas, un primer ánimo fáustico lo había arrojado al mundo del periodismo. De ese modo Park pudo dejar atrás el universo de la especulación, descender al mundo sensible e inmediato donde radicaba el valor de la experiencia (Dewey). La ciudad, una especie de organismo social a su parecer, se perfilaba igualmente como un microcosmos donde indagar y registrar puntualmente los avatares de la vida urbana (la pobreza, el vicio, los disturbios raciales, las epidemias, los tugurios, los establecimientos y trayectorias de los inmigrantes...). De esa manera podía obtener "una concepción de la ciudad, de la comunidad y de la región que iba más allá del simple fenómeno meramente geográfico" (Park 1950, viii).

Primero, en calidad de periodista:

"me dediqué a todo tipo de temas y conocí íntimamente una enorme cantidad de aspectos de la vida de una ciudad. Estoy seguro que cubrí muchos más kilómetros por las ciudades de todos los rincones del mundo que cualquiera de mis contemporáneos" (Ibid.). 
Como cuando en el New York Journal el city editor le envió a buscar un fumadero de opio y una casa de juego que la policía no terminaba de localizar.

"Entré en el fumadero de opio con mucha suerte (...). El lugar estaba atestado de la canalla de la ciudad y hablaban sin tapujos de la casa de juegos a la que yo pretendía acceder. Uno de ellos, ignorando quién era yo, consintió en conducirme hasta ella. Entramos pero no llegamos muy lejos. Uno de los dueños del 'garito' era bien célebre en los tribunales y, naturalmente, conocía a todos los reporteros habituales. Enseguida me reconoció y me sacaron fuera. ¿Si estaba asustado? A buen seguro que sí, pero ya tenía mi escrito" (Park, en Bourmeau 1988:51).

La experiencia periodística de Park le lleva por derroteros curiosos respecto a los temas de investigación sociológica convencionales y explica tanto su obstinación por la recolección de datos directos como su proceder (Faris 1969: 109). En realidad muchas de sus posteriores observaciones, los temas de las pesquisas que dirigió -sobre el comportamiento colectivo, la estructura de la ciudad y los tipos sociales que la habitaban (el hobo, los gangs, las taxis-dancers, el Jack-Roller...)- responden a un universo atípico para el académico pero familiar para el periodista de investigación. Una vez incorporados al campo de interés científico ganan cuerpo cuando son tamizados por la concepción sociológica de los procesos de desorganización social en la ciudad. Esta temática ha llevado a Roger A. Salerno (2007) a sugerir la precipitación de una sociología noire, (término aplicado a las narrativas y representaciones oscuras de la vida urbana), al modo en que hubo una novela negra y un cine negro, todos ellos productos culturales de un tiempo social y de unas vivencias específicas en la gran ciudad. (¡Se trata del Chicago de los roaring twenties!).

El interés por la observación directa, el trabajo de campo, el uso de mapas y otros tipos de representaciones cartográficas, las entrevistas, los estudios de casos e historias de vida también responden en gran parte al desempeño de su actividad como reportero. Esa curiosidad y esas artes de inmersión en la realidad social es lo que deseaba transmitir a sus alumnos, trabajando con ellos al modo de una redacción e incitándolos a bajar al terreno, en un ejercicio que prefigura el método del depth reporting. Anderson recuerda los consejos de Park para su investigación sobre The Hobo: "Anota todo cuanto veas, oyes y sabes, como un reportero", no dudando -a decir de Plenel (2008: 10)_ en construir esa ciencia de la sociología a partir de los "aportes bastardos e inclasificables como los del periodismo."

La preocupación por sustentar la explicación de los fenómenos sociales en la experiencia directa le llevó a insistir reiteradamente en la realización del trabajo de campo. Howard Becker, otro sociólogo poco convencional, se hace eco de las solicitaciones parkianas al respecto: 


\begin{abstract}
"Se os ha dicho que hojeéis en las bibliotecas y acumuléis notas y una gruesa capa de polvo. Se os ha aconsejado que elijáis los problemas de estudio que cuenten con amplia y enmohecida documentación, que repose en formularios preparados por burócratas hastiados y cumplimentados a regañadientes por aspirantes a una ayuda, por almas caritativas o empleados indiferentes. Es lo que se llama 'mancharse las manos con la auténtica investigación' (...). Pero es necesario algo suplementario: la observación de primera mano. Id a los salones de los hoteles de lujo, penetrad en los asilos nocturnos, sentaos en los canapés de la Gold Coast o en los jergones de los bajos fondos (...) En suma, ensuciaos los bajos de los pantalones en la verdadera investigación." (Chapoulie 2001: 118).
\end{abstract}

El trabajo de campo, aun con cierto aire de bricolaje y diletantismo, era un requisito formal de las monografías chicagüenses, pero sobre todo era una exigencia incluida en la formación sociológica de los estudiantes de Chicago. De hecho no era insólito verlos por diferentes áreas de la ciudad prestos a observar, anotar y describir cuanto sucedía, muchas veces acompañados por los profesores, Park entre ellos. Como éste afirmaba no era necesario dirigirse a regiones exóticas para encontrar contenidos de interés:

“(...) el hombre civilizado constituye un objeto de investigación igualmente interesante, y además su vida resulta más accesible a la observación y al estudio. La vida y la cultura urbanas son más variadas, sutiles y complejas, pero los resortes fundamentales son semejantes en ambos casos. Los mismos métodos de observación paciente que antropólogos como Boas y Lowie han aplicado al estudio de la vida y costumbres de los indios norteamericanos pueden emplearse incluso de forma más fructífera al estudio de las costumbres, creencias, prácticas sociales y concepciones generales de la vida que prevalecen en Little Italy, en el bajo North Side de Chicago, o para registrar los más sofisticados hábitos de los residentes de Greenwich Village y de los alrededores de Washington Square, en Nueva York" (Park [1915/1925]1999: 50)

Ciertamente encontramos un impulso original a los procedimientos etnográficos pero se trata de una etnografía atípica, casi una aproximación naturalista a las significaciones y a la dimensión subjetiva de los hechos sociales (Coulon 1994). Esta aspiración y su abordaje descansaban en la experiencia adquirida como reportero de investigación (teñida asimismo por un indisimulado reconocimiento por la literatura naturalista de Zola y Sherwood Anderson); también en la influencia pragmatista (que tendría continuidad en el interaccionismo simbólico); y por supuesto en los planteamientos de W. I. Thomas sobre la "definición de la situación". Había que situarse en la perspectiva del otro y ubicar dicha opción en el ámbito de los procedimientos empíricos, pues "el sociólogo no está interesado principalmente por los sucesos en sí mismos, los 
considera más bien como establecidos; le interesa especialmente las actitudes de las personas implicadas, tal como se reflejan en los muy diferentes relatos que ofrecen del mismo suceso histórico. Le interesa todo lo que pueda esclarecer las actitudes y hacerlas inteligibles" (Park, citado en Chapouile 2001: 117).

\section{INFORMACIÓN E INVESTIGACIÓN SOCIOLÓGICA}

"Cuando era city editor y reportero es cuando comienzo mis estudios de sociología... Para el artículo que escribí sobre la ciudad [The City, AJS, 1915] me apoyé mucho en la información que había adquirido como reporter observando la ciudad. Más tarde, cuando me correspondió dirigir un número cada vez mayor de estudiantes encontré que mi experiencia como city editor, dirigiendo un equipo editorial, me había puesto en una buena posición. La sociología, después de todo, trata sobre cuestiones a propósito de las cuales los periodistas tienen una buena dosis de conocimiento directo. Y junto a eso, la sociología trata precisamente de aspectos de la vida social que encuentran habitualmente sus expresiones más evidentes en las informaciones y documentos históricos y humanos. Se puede decir que un sociólogo es simplemente un reporter más científico, más preciso, más responsable" (Park en Bulmer, 1984: 91).

El sociólogo imaginado por Park se antojaba un súper-reportero. No hay en su planteamiento una ruptura epistemológica entre las praxis periodística y sociológica, sino tan sólo una diferencia de grado. La idea de un continuum aparece asimismo en la caracterización que Park realiza acerca del conocimiento intuitivo o familiar y el conocimiento científico en "News as a Form of Nowledge" (1940). Es típica de su esquema intelectual, donde prospera su inconfundible ambivalencia, que consiente interpretaciones compatibles: periodismo y sociología como ámbitos complementarios pero bien específicos. De ahí que la dedicación constante de Park para distinguir la observación sociológica de otras formas de escrutinio sea "algo que no está claro para él, que tiene un pasado periodístico del que no desea renegar y un nuevo estatus como sociólogo (...). Construye así una epistemología subyacente a sus trabajos (...) que puede ser leída en gran parte como una racionalización-legitimación de su propia trayectoria" (Bourmeau 1988: 59).

Lo que comparten ambos, el Park periodista y el Park sociólogo, es una lucha tenaz contra los enfoques morales, como proponía el movimiento reformista. En el periodismo la referencia es el muckraker (aunque en sus escritos sobre la sociología de la prensa sus consideraciones podrían aplicarse a los artículos que anteponen opinión a información); en la sociología, el peligro viene del movimiento de encuestas sociales. 
A finales del siglo XIX y principios del XX, el contexto social urbano generado por el capitalismo resultó tan desgarrador, tan excesivas las desigualdades, y la corrupción política y moral tan extendida que surgió un tipo de periodismo que orientaba su labor a la denuncia social. El muckraker era el héroe de tales hazañas ${ }^{4}$ : una figura consagrada al periodismo de investigación, al compromiso social, que advertía mediante sus escritos de las terribles condiciones de vida de los más humildes. El propio Park confiesa haberlo practicado $^{5}$ y llega a reconocerlo como una fuente no desdeñable de apreciaciones originales sobre la ciudad y el arte de la investigación, una vez pulido su tono dramático. No obstante, la sociología parkiana lo aparta desde el momento en que el propósito es avanzar por un conocimiento objetivo basado en la información precisa. De ahí que con el tiempo más que un modelo de investigación este tipo de investigación periodística sea tematizada como objeto de estudio/investigación (The Survey).

Ciertamente esta práctica de investigación cobró forma en el marco de un movimiento reformista más amplio que alcanzó incluso a la sociología americana en sus primeros pasos. En efecto, la primera generación de los sociólogos chicagüenses contemplaba la ciencia social como una herramienta de conocimiento e intervención sobre los problemas sociales. Esta disposición encontraba eco en las numerosas asociaciones cívicas y caritativas (Hull House, The Working People's Social Science Club, etc.) que instruían "encuestas sociales" en la órbita de la sociología aplicada y la asistencia social, procediendo en los social settlements de inmigrantes y desfavorecidos de la metrópoli. Park asumía el tributo debido al movimiento reformista en lo que se refiere a la investigación primaria. Empleó de hecho los pormenorizados estudios de Booth en Life and Labour of the People of London; se apoyó en la experiencia de los Hull House Maps and Papers en el curso de sus exposiciones sobre la desorga-

${ }^{4}$ Destacaron por su difusión e impacto The Shame of our Cities de Lincon Steffens (1904) a quien Park admiraba; Tramping with tramps, Studies and Sketches of Vagabond Life (Josiah Flynt, 1901); My Mamie Rose (O. Kildare, 1903), The Jungle (Upston Sinclair, 1905), así como How the other half lives de Jacob Riis. Bajo distintos formatos, siempre bien documentados en la medida en que son protagonizados por sus autores, estos escritos operarían en varios frentes: el político (reforma social), el profesional (periodismo) y el metodológico (estudio de caso).

${ }^{5}$ Aunque renegaba después de esta orientación, Park asumió este rol en el seno de la Congo Reform Association cuando se inicia la batalla internacional contra la barbarie del dominio belga en la colonia africana. Park publicaría en el Everybody's Magazine "The blood money of the Congo", "Recents atrocities in the Congo State", "The terrible story of the Congo", siempre en la órbita del reformismo y la práctica del muckraking. Lyman (1992) enjuicia estos textos como góticos, de tintes dramáticos, al uso de la época, repletos de lugares comunes que los redactores toman prestados de los relatos de los misioneros. 
nización social en la ciudad; y otro tanto puede decirse del Pittsburg Survey de P. Kellog, referencia mayor de la encuesta social. Apreciaba su interés y los datos que suministraban, pero les concedía un estatuto menor: no eran un modelo metodológico, sólo fuente de documentación e incluso objeto de estudio (así resultaba en el curso sobre The Survey. De la misma forma que consideraba que la información (de los hechos) convierte al reportero en un reformador más eficaz que el editorialista, también deseaba desmarcar la sociología del ánimo intervencionista, de los do-gooders, de los programas dirigidos de acción política y del trabajo social. Por eso desconfiaba del despliegue de los social surveys por cuanto la definición del objeto de estudio (los problemas sociales) y los métodos analíticos propuestos podían contaminar eventualmente la naturaleza científica de la sociología.

"Gran parte de la información se ha recolectado simplemente con el fin de determinar qué hacer en un caso dado. Los hechos no se han seleccionado para contrastar teorías sociales. Los problemas sociales se han recogido en su mayoría para apoyar tal o cual doctrina, no para probarla. En muy pocos casos se han hecho investigaciones, desinteresadamente, para determinar la validez de una hipótesis." (Park \& Burgess, 1921: 44).

La construcción de un ámbito de jurisdicción propiamente científico exigía otra definición del objeto y método sociológicos, afirmada sobre la neutralidad axiológica. Debía diferenciarse pues entre la investigación social y la investigación sociológica, neutral y objetiva. Y en ese sentido, insistía en que

"lo primero que han de aprender los estudiantes de sociología es a observar y a registrar sus observaciones (...) más que a formular opiniones. Los hechos más importantes de que tienen que ocuparse los sociólogos son opiniones (actitudes y sentimientos); pero en tanto que (...) no aprendan a tratar las opiniones como los biólogos tratan los organismos -es decir, disecándolas, reduciéndolas a sus elementos primarios, describiéndolas (...) no cabrá a obtener un progreso señalado de la ciencia sociológica" (Park, en Hughes 1974: 616)

Park activaba una orientación clínica de los procedimientos pertinentes de observación del cambio social y la naturaleza del conocimiento científico. El análisis de la sociedad no podía ser científico y moral a la vez, dos planos sobre la realidad social difícilmente reconciliables: el descriptivo y el normativo. Esta postura intelectual resultaba perfectamente válida para el ejercicio del periodismo, como se desprende de sus escritos sobre la prensa y de la experiencia misma del Thougts News (sobre el papel de las noticias y el influjo en la opinión pública de la información imparcial). 


\section{COMUNIDAD Y COMUNICACIÓN EN EL MEDIO URBANO}

La formalización de la perspectiva ecológica vino en auxilio de una determinación más clara de la objetivación y los procedimientos clínicos, aspirando a la búsqueda de generalidades sobre el desarrollo de la sociedad desde las regularidades observadas en el metabolismo social y en los procesos implicados. Es el paso necesario para elevar hipótesis relativas al ámbito de la superestructura moral de la existencia colectiva. Así, la ecología humana parkiana estimaba (como antes la morfología social durkheimiana) que el incremento del volumen y densidad de la población acrecentaba la división del trabajo, la diferenciación social y los procesos de individualización, con sus implicaciones tanto en la estructura territorial de los grupos sociales como en el desarrollo mismo de las interacciones sociales y de un nuevo orden moral.

Remitiéndonos a la articulación entre los procesos ecológicos y culturales, el modelo metropolitano de concentración y diferenciación interna de las actividades económicas, grupos sociales y hábitats residenciales (basado en la competencia biótica, el dominio y la sucesión) resulta mediado por la comunicación en el plano material (vías, medios e instrumentos) y en el social (interacciones, representaciones colectivas, opinión pública). Sin los modernos medios de comunicación sería harto complicado comprender la transformación de la metrópoli. Los medios acentúan la concentración y la movilidad en un proceso de implosión-explosión general y de localización diferencial (distritos comerciales, suburbios residenciales, áreas naturales), ambas inscritas en una urbanización de los valores, hábitos y estilos de vida. La sociedad sólo existe en y es posible por la comunicación, que habilita la integración social de los elementos de un conjunto diversificado pero interdependiente como la metrópoli moderna. Concilia la posibilidad de la vida individual en el marco de una existencia colectiva. Puede aventurarse, pues, un orden moral instituido sobre una base ecológica; dicho de otro modo, sobre la infraestructura biótica (basada en la competencia) se constituye una superestructura moral (basada en el consenso, la tradición y la costumbre). Un orden moral en continuo ajuste, dinámico y precario no obstante, pues la metrópoli representa una sociedad en transición permanente hacia formas articuladas de solidaridad orgánica. Como realidad heterogenética y compleja por su tamaño, densidad, diversidad étnica y profesional, la gran ciudad define una nueva forma de existencia: nuevos patrones de interacción social, de comportamiento y de organización. Un ambiente caracterizado por una potente carga secularizadora y por un racionalismo imposible de hallar en las pequeñas comunidades, apegadas al ámbito local y a lo concreto, sometidas al ciclo repetitivo de ritmos e interacciones. 
Asociada a la idea de comunicación -por su capacidad para neutralizar las distancias físicas y sociales- es imposible eludir la importancia atribuida a la movilidad, especialmente sus efectos en el despliegue de disposiciones típicas del temperamento urbano: el pensamiento abstracto y el cosmopolitismo. Por lo demás, la movilidad aparece inscrita en los ciclos de organización-desorganización-reorganización que atraviesan grupos e individuos en diferentes momentos de su desarrollo. En "La comunidad urbana como modelo espacial y orden social" (1926) Park es categórico al respecto:

"El telégrafo, el teléfono, la prensa y la radio transforman el mundo en una gigantesca cámara de resonancia, anulando las distancias y quebrando el aislamiento que antes separaba a razas y pueblos. Nuevos medios de comunicación están permanentemente multiplicando y complicando las relaciones sociales. La historia de la comunicación es, en un sentido propio, la historia de la civilización. (...) Pero, hay que decirlo, los medios de comunicación no tendrían la significación que han adquirido hoy en día si su desarrollo no hubiera estado acompañado de un incremento de la división del trabajo. (...) Precisamente porque la comunicación desempeña un papel fundamental en la existencia de una sociedad puede decirse que la geografía y todos los demás factores que limitan o facilitan la comunicación forman parte de su estructura y de su organización. (...). Como concepto sociológico, la movilidad sólo es significativa en la medida en que asegura nuevos contactos sociales, y la distancia física sólo tiene valor para las relaciones sociales en tanto que es posible interpretarla en términos de distancia social. (Park [1926] 1999: 97-98).

Se trata de una movilidad territorial que supone necesariamente el cambio de perspectivas y de referencias en un nuevo entorno. Pero la movilidad no se limita al cambio de localización; también puede medirse "por el número y la diversidad de estímulos a los que uno y otra responden. La movilidad depende no sólo de los transportes sino también de la comunicación". (Park [1926] 1999: 97). Estamos ante una segunda modalidad de movilidad típicamente social -de posiciones sociales en la estructura ocupacional y cultural-. Y puede ser una movilidad con o sin desplazamiento físico. La ciudad es precisamente el ámbito privilegiado de posibles e incesantes interacciones entre grupos e individuos que la habitan, usan y construyen, lo que marca el acceso a diferentes sistemas de significados, a nuevos modos de obrar y pensar. Se trata de la experiencia de la alteridad y lo que conlleva: universos ajenos de discurso en situación de co-presencia, diferentes referencias sociales al margen de las constricciones del grupo primario (familia, vecindario, localidad).

Llevada a sus últimas consecuencias, la argumentación sobre la movilidad en "La ciudad" describe una realidad social bifronte: cuanto posee de estimulación intelectual lo tiene de inestabilidad y fragilidad. 
"Los transportes y las comunicaciones han provocado, entre otras modificaciones silenciosas pero profundas, lo que he denominado 'la movilización del individuo'. (...) han multiplicado para el individuo las oportunidades de contacto y de asociación con sus semejantes, pero han vuelto esos contactos y las relaciones más efímeras e inestables. Una gran parte de la población de las grandes ciudades, incluidos los que residen en apartamentos o en casas de vecindad, viven más bien como si de un hotel se tratase, encontrándose pero sin conocerse entre ellos. De ahí deriva que las relaciones formales y ocasionales sustituyan a las estrechas y permanentes relaciones de la pequeña comunidad" (Park [1915/1925], 1999: 79).

Las mutaciones en la estructura material y social de la gran ciudad conllevan, como proceso crítico, fases de desorganización social y de desintegración moral de los individuos. La ruptura de los vínculos primarios, de las ataduras locales, de sus inhibiciones, la pérdida de influencia por parte instituciones primarias, la movilidad extrema..., todo eso puede llegar a provocar la quiebra del control social tradicional y la aparición de formas de comportamiento desviado. Muchas de las investigaciones de Chicago siguen esa dirección: los procesos de aculturación y desintegración moral en la ciudad (esa sociología noire a la que antes se aludía). Sin embargo, los cambios habidos introducen un nuevo orden que contiene las pulsiones del hombre y los comportamientos livianos. Independientemente de que la ley sustituya a la costumbre, el control social adopta una forma indirecta pero no menos efectiva: la moda y la opinión pública (el rumor de la aldea es sustituido por la prensa). La comunicación, al permitir una acción concertada, se erige como instrumento de cohesión social y acomoda el desarrollo individual al colectivo.

\section{DONDE LA CIUDAD SE ESCRIBE: CONSIDERACIONES SOBRE LA PRENSA EN EL MEDIO URBANO}

El interés por el orden y el cambio social en la ciudad, auspiciados por la comunicación, resulta patente en la sociología de la prensa parkiana. ¿No es acaso la prensa un producto típicamente urbano, como observa la historia de las ideas y de la publicidad? Park indaga en esta vinculación en textos como "Natural History of Newspapers" (1923), "The immigrant community and immigrant press" (1925), "Urbanization as measured by newspaper circulation" (1929), "Newspaper circulation and metropolitan regions" (1933). Ya no es algo transversal, sino un estudio franco sobre el vínculo (1) entre prensa y urbanización, entendida ésta como proceso de extensión, articulación y concentración de actividades y población heterogénea en el espacio; y (2) entre prensa y urbanismo, entendido éste como un modo de vida, lo que discurre por una 
caracterización típicamente cultural de ambos fenómenos. El interés de esos artículos radica en el modo original como Park aborda la evolución de la prensa a partir de la perspectiva ecológica. Por supuesto hay otros ecos, entre ellos los simmelianos -sobre la historia natural de las instituciones- pero resulta notable la forma en que esos procesos sin sujeto típicos del darwinismo y la ecología de Haeckel (las leyes de la competencia biótica y sus derivados, la sucesión, la dominación, la adaptación) esclarecen la transformación de este producto cultural que es la prensa en el mundo contemporáneo. Un mundo que es sobre todo urbano, de ahí la analogía empleada en la apertura del artículo:

"La prensa, como la ciudad moderna, no es una creación totalmente racional. Nadie ha buscado hacer de ella exactamente lo que es. Pese a todos los esfuerzos de individuos y generaciones enteras por controlarla y moldearla su manera, ella continúa creciendo y evolucionando según sus propias vías imprevisibles" (Park 2008, 41).

Dos cuestiones destacan en el planteamiento:

1. Prensa y urbanización discurren unidas. La transformación y extensión de la estructura urbana se asocia a la transformación de los medios (contenidos, formatos, fines y actuaciones).

2. La declarada naturalidad del fenómeno establece de un lado un ámbito de jurisdicción y metodología científica propia (la prensa como objeto de estudio clínico, desapasionado); de otro, la inequívoca pretensión de desvincular la "naturaleza" y evolución propia de la prensa respecto de las lecturas moralistas, del tipo The Brass Check de Sinclair. Park elude esta caracterización para tomarla como un producto de las condiciones de la vida moderna, de lo que se desprende un recorrido en términos evolutivos.

"Natural History of Newspaper" (1923), artículo incorporado en The City (1925) es ejemplar en ese sentido: explora la evolución de la prensa al hilo de las mutaciones de la estructura física, sociodemográfica y cultural de la ciudad. Atendiendo a esta contextualización, Park distingue distintas tipos de prensa.

- La primera forma no es sino una mera continuación rústica de los newsletters. El Boston Newsletter, primer periódico americano, surge en la estafeta de correos, lugar de encuentro y debate de los sucesos nacionales y comunitarios. "Si algún periódico debía ver la luz ése era el lugar idóneo, en proximidad inmediata de las fuentes de información" (Park 2008, 45). Marca una tendencia en la prensa local: el interés por el mundo próximo, y la organización del cotilleo y el rumor característicos de la vida 
de las pequeñas comunidades. Instrumentos de control social y a la vez de cohesión, la prensa amplificará su alcance bajo formatos de crónica costumbrista aderezada con aires novelescos.

- El segundo tipo se vuelca en la opinión. Tenía un antecedente en el registro de los debates parlamentarios de las primeras democracias liberales. Su recorrido es amplio y pone de manifiesto las relaciones de la prensa con el poder así como el poder de la prensa, que como apunta el propio Park -antes de que esta teoría sea elaborada- termina imponiendo la agenda a los políticos ${ }^{6}$. Esta prensa de opinión, que deriva hacia prensa de partido, tiene su apogeo en los EE.UU. durante la campaña antiesclavista (New York Tribune).

- Sacudiéndose paulatinamente la presión y dependencia de los partidos, surge y crece en las grandes ciudades la prensa independiente (v.gr. New York Times), de base comercial, retomando la importancia de los hechos y la difusión de las noticias. Es en la información y no en las doctrinas donde radica la virtud de la prensa, proporcionando referencias.

- El tipo de periódico que se impone en el ámbito metropolitano masificado es el correspondiente a la prensa sensacionalista, mezcla de literatura e información, jugando con las emociones, amor y romance para las mujeres, y el deporte y política para los hombres. Receta aplicada con éxito por J. Pulitzer (New York World) y William R. Hearst (San Francisco Examiner).

La evolución de la prensa sigue los patrones ecológicos asociados a los procesos de sucesión y dominación derivados de la competencia por la difusión; y es tributaria del progreso de la ciudad, igualmente regida por dichos procesos ecológicos. Hay que tener presente el incremento de lectores en el medio urbano pues con el advenimiento de la sociedad de masas - una sociedad compleja con un alto grado de diferenciación social y de división del trabajo- se impone la prensa con mayor capacidad de penetración entre el público urbano, al que proporciona referencias prácticas bajo un producto adaptado, normalizado y de registro cómodo. "Donde todo sucede todos los días no es posible dar detalles de cada uno de los incidentes, de las cosas fuera de lo común o de la rutina, pero se pueden seleccionar ciertos incidentes particularmente sorprendentes o novelescos para tratarlos como símbolos, por su dimensión

${ }^{6}$ Weber (1910) recuerda como en el siglo XVIII la prensa debía arrodillarse y pedir perdón ante el Parlamento inglés por violar el privilegio de conocer las deliberaciones del Parlamento (breach of privilege). En 1910 es la prensa la que pone de rodillas al Parlamento con la sola amenaza de no publicar sus discursos. Ese poder irá más lejos, como señala Park, adelantando teóricamente la importancia de la agenda mediática. 
humanamente ejemplar más que por su importancia individual y singular. La información en la ciudad pierde su carácter personal para hacerse arte. Deja de ser el relato de las actuaciones de hombres y mujeres concretos para convertirse en la crónica impersonal de costumbres y de la vida" (Park 2008, 47).

Esto no es óbice para que en el medio local sean bien recibidas las noticias sobre su entorno. Los periódicos locales inciden en esta faceta, que procura conocimientos prácticos y sociales necesarios (como aún hacen los diarios gratuitos o secciones locales de la prensa nacional). Pero la dominación de las grandes ciudades, la influencia que ejerce sobre su entorno es un hecho que ya no puede dejarse de lado, y los periódicos de vocación metropolitana constituyen una herramienta de propagación de esta dominación urbana. He aquí una evaluación cultural de la prensa metropolitana en tanto que instrumento de socialización y homogeneización, extendiendo el universo de los valores urbanos.

La circulación de la prensa resulta incluso un buen indicador de la dominación de la cultura y economía urbanas. Park explora esta vinculación, primero en "Urbanization as measured by newspaper circulation" (1929) y después, en una escala ampliada, en "Newspaper circulation and metropolitan regions" (1933). Basándose en el concepto de área cultural de Galpin y haciendo uso de la cartografía, se observa que "la circulación de la prensa, cuando es punteada en un mapa, sirve para delimitar con excepcional certeza los límites del área comercial local, y para medir al mismo tiempo la extensión y grado de dependencia de los suburbios respecto a la metrópoli, y de la metrópoli respecto a la región más extensa que domina" (Park 1974, 378). La urbanización ha quebrado los límites de la ciudad concebida como una estructura material coherente y contenida. El tejido urbano adopta una forma dispersa, dando lugar a suburbios residenciales entremezclados con usos rurales, industriales y comerciales. La extensión material y cultural de la ciudad se puede estimar indirectamente mediante la circulación de la prensa, en un gradiente de círculos concéntricos, similar al de los valores del suelo: desciende regularmente desde el centro hacia la periferia.

El análisis de la circulación de la prensa pone de manifiesto que:

1. la definición administrativa de ciudad resulta superada por el dinamismo de su realidad económica, que impone una lectura de las interrelaciones funcionales con el entorno;

2. la proliferación de estilos de vida diferenciados, como en los suburbios. Sus habitantes mantienen una vinculación intensa con el centro de mercado, posiblemente más que con su entorno inmediato. (Se trata de la commuter zone, el hábitat de los trabajadores pendulares, de alta movilidad social y espacial). 
Si el hábito y tipo de lectura de prensa proporciona un indicador indirecto sobre la cultura suburbana, otro tanto puede decirse de las subculturas urbanas. La variedad ecológica y social de la gran ciudad no se muestra únicamente en sus bordes, pues su configuración espacial exhibe áreas internas más o menos segregadas donde habitan grupos sociales de distinto rango y origen étnico. Park prestó una atención especial a estas áreas de inmigración. Chicago, esa ciudad de frontera, contaba con sectores como el Ghetto, Little Sicily, Black Welt, etc. que ofrecían un mosaico colorido de sectores diferenciados por sus moradores, costumbres, valores, universos de discurso, normas de decencia y presentación. Pero sectores sometidos también a la descomposición de los vínculos sociales primarios en la vorágine de la metropolización. Fuera del control ecológico del grupo, el individuo posee la libertad de moverse por los diferentes ambientes y regiones morales, dejando atrás las constricciones del grupo primario. Es el reverso de la desestabilización social, la desorganización grupal asociada a la movilidad colectiva e individual: ruptura de las continuidades históricas o de la reproducción del grupo. Situación típica en las comunidades inmigrantes que sufren la tensión de una identidad de origen (primera generación) y otra de destino (segunda generación). Es la tensión de los dobles vínculos, que se recrudeció en la época en que América se disponía a entrar en la Gran Guerra, planteándose el problema de la naturalización o "americanización de las poblaciones" ("the strangers within our gates").

Retomando la digresión simmeliana sobre el extranjero, Park veía en el inmigrante no sólo al sujeto concreto sino el personaje conceptual capaz de expresar el juego dialéctico de la proximidad y la distancia (proximidad espacial, distancia anímica) típica de la modernidad metropolitana: los vínculos sociales se despliegan en un juego entre la proxemia y la diastemia, acentuado con la división del trabajo, la diferenciación social y el individualismo. Pero la prensa contribuyó en parte a la asimilación de estas comunidades. En Nueva York llegó a haber 270 publicaciones sostenidas mayoritariamente por la población local, impresas en 23 lenguas diferentes. Y en Chicago hubo 19 diarios publicados en 7 lenguas extranjeras con una tirada diaria de 368.000 ejemplares. Remitiéndose a The Polish Peasant de Thomas \& Znaniecki, Park rescata la declaración del jefe de redacción del periódico publicado en ruso, Ruskoye Slovo de Nueva York, según el cual, tras una encuesta entre sus lectores encontró que de 312 interrogados sólo 16 leían regularmente el diario en Rusia y 10 de vez en cuando, y sólo 12 abonados a un semanal en su tierra de origen; alguno leía la hoja de la administración (volast). Sin embargo, trasladados a Nueva York todos pasaron a lectores asiduos de periódicos y revistas. Si la lectura era "un lujo en el campo, se convierte en una necesidad en la ciudad. En el medio 
urbano saber leer es casi tan indispensable como saber hablar. Esa es una de las razones de la abundancia de diarios en lengua extranjera" (Park 2008, 42). La prensa local actuó como agente de socialización proporcionando referencias grupales mientras abría una ventana a un mundo próximo más amplio, acompañando las incursiones de estas poblaciones hacia el exterior de sus enclaves donde hábitos, lengua, normas y valores eran desconocidos. Les suministró en suma referencias generales de la vida en las grandes ciudades americanas, mostrando que la urbanización tenía a la vez un componente cultural.

\section{CONCLUSIONES}

Regresar a la obra y trayectoria de Robert Park contribuye sin duda a superar la célebre desconfianza, aún bien patente, entre la sociología y el periodismo. Park, que reivindicó su pasado como reportero, concebía al sociólogo como un super-reportero. El estudio de la ciudad, que ha marcado ampliamente su recepción, se alimenta de hecho de la experiencia adquirida en el periodismo de investigación. Así, todo cuanto había aprendido sobre la ciudad en el curso de sus reportajes urbanos, la comparación entre las ciudades que había conocido durante sus quehaceres, la importancia atribuida a la comunicación como urdimbre social, su consideración sobre la objetividad de las informaciones recolectadas y trasmitidas, o el riesgo de propagar opiniones y posiciones morales son afluentes imprescindibles para comprender la contribución parkiana al estudio de la ciudad, a la metodología de la investigación sociológica y a esa temática noire a la que aludíamos.

"La ciudad" (1915/1925), texto que inaugura de hecho su orientación hacia la sociología urbana, no es concebible sin dicha referencia: tanto en el texto como en la realidad descrita los procesos urbanos y comunicativos se manifiestan necesaria y estrechamente vinculados. Esta ligazón se expresa asimismo en otros muchos escritos "urbanos" donde aborda la influencia de la comunicación y de los medios en la configuración material de la ciudad o en aquellos otros donde examina el papel de la prensa en la orientación de la cultura urbana moderna. Porque la urbanización nunca es vista como mera aglomeración de actividades, grupos sociales e individuos sobre un punto sino como un proceso cultural, que da lugar a comportamientos novedosos, a patrones normativos innovadores y a estilos de vida o tipos sociales característicos. La prensa moderna, una creación de esas condiciones urbanas, contribuye a la difusión de estos valores urbanos, acelerando además el proceso de asimilación entre los urbanitas de toda condición y origen. De ahí que se le conceda un papel básico en la definición de un nuevo orden social, característico de entornos complejos donde las relaciones secundarias cobran importancia; y en esa misma medi- 
da, un papel socializador, especialmente para los inmigrantes. Estos siempre requieren instrumentos que faciliten el recuerdo en común, las referencias tradicionales, un grado de privatismo. Su organización familiar y los protocolos de interacción interna cumplen durante un tiempo tal cometido. Pero fuera del vecindario, el espacio público urbano es el área de contactos secundarios donde las relaciones son relativamente impersonales, ofreciendo un registro de socialización más amplio y plural, como el del espacio de la publicidad y la prensa.

\section{REFERENCIAS BIBLIOGRÁFICAS}

Abbot, A. (1999). Department and Discipline, Chicago School Sociology at One Hundred. Chicago: The University of Chicago Press.

Berganza Conde, R. (2000): Comunicación, opinión pública y prensa en la sociología de Robert E. Park, Madrid: CIS.

Bourmeau, S. (1988). "Robert Park, journaliste et sociologue", Politix, 1, nº 3-4, 50-61.

Breton, D. Le (2008). "Park et l'interactionnisme symbolique", en GUTH (dir.) Modernité de Robert Park, 261-270.

Bleman, L. S. (1976). "Robert E. Park: An Intellectual Portrait of a Journalist and Communication Scholar". Journalism History, vol. 2, 4, 116-132.

Blumer, M. (1984): The Chicago School of Sociology: Institutionalization, Diversity and the Role of Sociological Research, Chicago: University of Chicago Press.

Chapoulie, J.M. (2001). La tradition sociologique de Chicago (1892-1961), Paris: Seuil.

Cefaï, D. (2008). "Vers une écologie des publics. Park, l'opinion publique et le comportement collectif", en GUTH, op. cit., 155-188.

Coulon, A. (1994). L'École de Chicago. París: PUF (2ª ed.), 1994.

Faris, R. E. (1967). Chicago Sociology 1920-1932, Chicago: University of Chicago Press. Grafmeyer, Y. \& Joseph, I. (1979). L'École de Chicago. Naissance de l'ecologie urbaine, París: Aubier.

Guth, S. (2008) (dir.) Modernité de Robert E. Park, Paris: L'Harmattan, 313 p.

- (2012). Robert E. Park. Itinéraire sociologique de Red Wing à Chicago. París: L'Harmattan. Halbwachs, M. (2008): "Chicago, experiencia étnica", en MARTINEZ, E. (ed. y trad.) Maurice Halbwachs: Estudios de morfología social de la ciudad, Madrid: CIS.

Lannoy, P. (2004). "Quand Robert Park écrit 'La Ville' (1915). Essai de scientométrie qualitative". Revue d'Histoire des Sciences Humaines, 11, 157-184.

Lazar J. (2006). "Quand les nouvelles étaient une forme de connaissance. Souvenir insolite d'Ezra Park". Géographie, Économie, Société, 8, 489-498

Lindner, R. (1996). The reportage of urban culture- Robert E. Park and the Chicago School, Cambridge, Cambridge University Press.

Lyman, Stanford. M. (1992). Militarism, Imperialism and Racial Accommodation. An Analysis and Interpretation of the Early Writings of Robert E. Park, Fayeteeville: University of Arkansas Press.

Martínez, E. M. (1999): "Introducción", en PARK, R.E., La ciudad y otros ensayos de ecología urbana, Barcelona: Serbal, 7-47. 
- (2013). "La investigación ecológica de las comunidades locales". Empiria. Revista de Metodología de Ciencias Sociales, 25, 173-194.

Park, Robert E. (1922). "The Immigrant Press and its control", Americanization studies, the acculturation of immigrant groups into American society, Harpers and Brothers, 1992, cap. 16 (vol. 7)

- (1950-1955). Collected Papers of R.E. Park, Everett C. HUGHES et al., Glencoe, Ill: Free Press.

- (1974): "La urbanización medida por la circulación de prensa", en THEODORSON, Estudios de Ecología Humana, Barcelona: Labor, 1974, 377-390.

- (1999), La ciudad y otros ensayos de ecología urbana, Barcelona: Serbal (trad. y ed. de E. Martínez)

- (2008). Le journaliste et le sociologue (ed. por G. Mulhmann y E. Plenel), París, Le Seuil Park, R. y Burgess, E. (1921). Introduction to the Science of Sociology, Chicago: The University of Chicago.

Plenel, E. (2008). "Actualité de Park", en Park, R. Le journaliste et le sociologue, 7-18. Raushenbush, W. (1979) Robert E. Park. Biography of a sociologist, Durham: Duke University Press.

Salerno, R. (2007). Sociology noir. Studies at the University of Chicago in loneliness, marginality and deviance, 1915-1935. Jefferson (N.C.) MacFarland and Co. Inc. Publisher.

Sánchez de la Yncera, I. y López-Escobar, E. (1996). "Los barruntos de Park. Antes de Chicago". Revista Española de Investigaciones Sociológicas, 74, 345-359. (Incluye la traducción de La masa y el público de R. Park, 361-423).

Weber, Max (1910). "Para una sociología de la prensa", REIS, 57, 1992, 251-259. Weber, Marianne (1995). Max Weber. Una biografía. Valencia: Alfons el Magnanim.

EMILIO M. MARTÍNEZ GUTIÉRREZ es profesor titular de Sociología en la Universidad Complutense de Madrid. Anteriormente fue profesor en la Universidad de Alicante e investigador del Instituto Multidisciplinar de Estudios del Medio Ramón Margalef (UA), profesor visitante en la Universidad de París V (René Descartes) y del Cente Max Weber (Lyon II). Su área de trabajo principal versa sobre temáticas urbanas, sobre las que ha publicado numerosos artículos y libros así como traducciones de autores clásicos (R.Park, Wirth, H. Lefebvre y Maurice Halbwachs).

Recibido: 19/02/2016

Aceptado: 29/06/2016 